\title{
O período da contaminação com petróleo influencia a rebrota de Echinochloa polystachya (H.B.K.) Hitchcock em solo de várzea da Amazônia Central?
}

\author{
Aline Lopes ${ }^{1,3}$ \& Maria Teresa Fernandez Piedade ${ }^{2}$ \\ ${ }^{1}$ Instituto Nacional de Pesquisas da Amazônia, Coordenação de Pesquisas em Ecologia, \\ Grupo Maua (Projeto INPA/Max-Planck) \\ ${ }_{2}^{2}$ Instituto Nacional de Pesquisas da Amazônia, Coordenação de Pesquisas em Biologia Aquática, \\ Grupo Maua (Projeto INPA/Max-Planck) \\ ${ }^{3}$ Autor para correspondência: Aline Lopes, e-mail: alopesmga@gmail.com
}

LOPES, A. \& PIEDADE, M.T.F. The period of contamination with petroleum influences the regrowth of Echinochloa polystachya (HBK) Hitchcock in varzea soil in Central Amazon? Biota Neotrop. 10(4): http:// www.biotaneotropica.org.br/v10n4/en/abstract?article+bn02910042010.

\begin{abstract}
Several factors may influence the impact of oil on the environment. However, although it is understood that the effect of pollutants may change throughout the year according to seasonal variations in environmental parameters, this effect is poorly studied in the tropical region. The effects of Urucu's crude oil on the vegetative propagation and growth of Echinochloa polystachya were evaluated in a 63 days period, in two experiments, "A" (July-September) and "B" (September-November) planting the species in a greenhouse. In both experiments parts of stems were placed in $2 \mathrm{~L}$ of várzea soil contaminated by 6 oil doses, ranging from 0 to $0.231 \mathrm{~L} \mathrm{oil} \mathrm{m}^{-2}$ soil. In response to dosage increase there was a decrease of total biomass, ratio of live /total biomass, the leaf length and number of leaves. The period of planting influenced the response of plants to the dosage applied due to climate change, with negative effects in the " $\mathrm{B}$ " period of higher temperatures. We concluded that the exposure period influence the vegetative propagation and growth of the seedlings, being a spill in the period of the higher temperatures more dangerous for this specie.
\end{abstract}

Keywords: floodplains, aquatic herbaceous plants, light petroleum, Urucu.

LOPES, A. \& PIEDADE, M.T.F. O período da contaminação com petróleo influencia a rebrota de Echinochloa polystachya (H.B.K.) Hitchcock em solo de várzea da Amazônia Central? Biota Neotrop. 10(4): http://www. biotaneotropica.org.br/v10n4/pt/abstract?article+bn02910042010.

Resumo: Diversos fatores podem influenciar o impacto do petróleo no meio ambiente. No entanto, embora seja sabido que o efeito dos poluentes pode mudar ao longo do ano de acordo com as variações sazonais em parâmetros ambientais, esse efeito é pobremente estudado na região tropical. Foi estudado o efeito do petróleo de Urucu sobre o rebrotamento e crescimento de Echinochloa polystachya durante 63 dias, em plantios estabelecidos em casa de vegetação em dois períodos do ano, "A" (julho a setembro) e "B" (setembro a novembro). Os propágulos foram plantados em frascos com 2 L de solo contaminado pela mistura de 6 dosagens de petróleo, distribuídas entre 0 e 0,231 L óleo $\mathrm{m}^{-2}$ de solo. Em resposta ao aumento da dosagem do petróleo houve diminuição da biomassa total, da biomassa aérea viva/total, da biomassa aérea, do comprimento das folhas e do número de folhas. O período de plantio influenciou a resposta das plantas à dosagem aplicada o que foi positivamente correlacionado a alterações climáticas, com efeitos negativos acentuados no período "B", de temperaturas mais elevadas. Pode-se concluir que o período de exposição influencia a propagação vegetativa e crescimento das plantas jovens, sendo um derrame no período de temperaturas mais altas mais prejudicial para essa espécie.

Palavras-chave: áreas alagáveis, plantas herbáceas aquáticas, petróleo leve, Urucu. 


\section{Introdução}

As atividades de extração, transporte e refinamento de petróleo têm contribuído para a contaminação do solo com hidrocarbonetos de petróleo em todo o mundo. Apesar dos grandes derramamentos gerarem comoção pública pelos danos causados, eles contribuem pouco, cerca $10 \%$, para a percentagem total de poluição do solo e da água (Dowty et al. 2001). De acordo com a Petrobrás (2007) os vazamentos de petróleo e derivados no Brasil totalizaram $386 \mathrm{~m}^{3} \mathrm{em}$ 2007, sendo registradas 86 ocorrências no total. Na região amazônica, onde o transporte do petróleo é feito por oleodutos e navios petroleiros por cerca de $650 \mathrm{~km}$, desde o local de extração até a refinaria (Val \& Almeida-Val 1999), o risco de contaminação das áreas alagáveis na bacia do Solimões/Amazonas é alto.

Diversos fatores podem influenciar o impacto do petróleo sobre o ambiente. Estudos em regiões temperadas sugerem que a sazonalidade climática influência o impacto do óleo na vegetação (Zengel \& Michel 1996). A sazonalidade climática pode afetar também os processos de recuperação, pois as plantas são mais sensíveis à limpeza do óleo no período de crescimento que durante a pré-dormência e dormência (Pezeshki et al. 2000). Durante a estação de crescimento os processos metabólicos nas plantas são intensos e fundamentais para o bom desenvolvimento do indivíduo adulto (Taiz \& Zeiger 2004) e, sob tais circunstâncias, o estresse da exposição ao petróleo pode levar à interrupção ou ao comprometimento irreversível de funções fisiológicas vitais (Pezeshki et al. 2000, Lopes 2007).

De acordo com Junk et al. (1989) a vegetação das áreas alagáveis amazônicas é estruturada pelo pulso de inundação. Na Amazônia Central o nível da água varia em média $10 \mathrm{~m}$ entre a fase aquática e terrestre, provocando o alagamento por aproximadamente 6 meses das cotas mais baixas do relevo, onde ocorrem diversas espécies da família Poaceae, dentre as quais se destaca Echinochloa polystachya (H.B.K.) Hitchcock pela grande quantidade de biomassa gerada anualmente, em torno de 108 t.ha $^{-1}$ em um ciclo anual de crescimento (Piedade et al. 1991). A biomassa de E. polystachya, distribuída em $65 \%$ de caules, 30\% de folhas e 5\% de raízes (Piedade 1988), é consumida por capivaras e algumas tartarugas; as folhas servem à alimentação de invertebrados terrestres e as raízes proporcionam substrato para invertebrados aquáticos e hábitat para peixes jovens (Piedade et al. 1992). Devido aos altos valores de produtividade primária produzida pela espécie ela é considerada fundamental para o ecossistema de várzea. Estudos prévios mostram que a concentração de elementos químicos é muito semelhante entre as diferentes partes do colmo e entre os períodos do ano, de tal forma que cada propágulo vegetativo tem proporções adequadas e equiparáveis de nutrientes para o seu rebrotamento (Piedade et al. 1992, 1997). A sazonalidade imposta pelo pulso de inundação implica que espécies como E. polystachya, que rebrotam durante a fase terrestre, poderiam ser fortemente afetadas por um derramamento de petróleo nesse período, com consequências mais graves do que aquelas da fase aquática, quando as plantas são adultas (Lopes \& Piedade 2009).

Este estudo foi desenhado para determinar se a capacidade de rebrotamento e o crescimento inicial de E. polystachya são influenciados pelo período do ano no qual a contaminação por petróleo ocorreu, verificando o efeito da dosagem de petróleo e a influência da temperatura do período de plantio.

\section{Materiais e Métodos}

As plantas foram coletadas na Ilha da Marchantaria $\left(03^{\circ} 15^{\prime} \mathrm{S}\right.$ e $060^{\circ}$ 00’ W), no médio Rio Amazonas, Brasil. Foram realizadas duas coletas (julho e setembro de 2006), de forma a propiciar o desenvolvimento das plantas com tratamentos experimentais em condições climáticas distintas de temperatura (Tabela 1). Foram utilizados os dados climáticos do Aeroporto Internacional de Manaus que possuem uma tendência muito similar aos coletados na estação climática do INPA onde os experimentos foram realizados, e cujos dados não estavam disponíveis durante todo o período experimental ( $\mathrm{r}=0,871, \mathrm{p}<0,001$; correlação de Pearson). No período "A", que compreendeu de 01 de julho a 01 de setembro, a temperatura ambiente máxima foi de $34,5^{\circ} \mathrm{C}$ no dia 29 de agosto e a mínima de $22,8^{\circ} \mathrm{C}$ no dia 31 de julho. No período "B", de 06 de setembro a 07 de novembro, as temperaturas foram mais elevadas com a máxima de $36,2{ }^{\circ} \mathrm{C}$ no dia 27 de setembro e a mínima de $23,4{ }^{\circ} \mathrm{C}$ no dia 2 de novembro (Tutiempo.net 2006).

Os experimentos foram realizados na Casa de Vegetação do Projeto INPA/Max-Planck, Manaus, Brasil. As plantas foram irrigadas diariamente com água proveniente de poço artesiano. O solo de várzea (70,5\% de argila, $17,92 \%$ de silte, $8,97 \%$ de área grossa, 2,61\% de areia fina; Lopes 2007) utilizado no plantio dos propágulos foi coletado em partes não inundadas da mesma ilha no mesmo período da coleta das plantas. Foi utilizado petróleo proveniente da Base Petrolífera da Petrobrás em Urucu, na Bacia Sedimentar do Solimões, Amazonas, Brasil. Este petróleo é considerado leve com grau API 45,8 (ANP 2008). Detalhes sobre a composição deste petróleo podem ser encontrados em Lopes et al. (2009). As dosagens de petróleo foram escolhidas por meio de um estudo prévio com dosagens de até $15,9 \mathrm{~L} \mathrm{~m}^{-2}$ de solo (Lopes et al. 2005).

Foram coletadas plantas adultas, das quais foi cortada uma parte do colmo de $15 \mathrm{~cm}$ contendo um nó. Estes propágulos foram plantados em bandejas plásticas $(27,0 \mathrm{~cm}$ de largura, 40,0 de comprimento e 7,0 cm de profundidade) com $2 \mathrm{~L}$ de solo que foi previamente contaminado pela adição de petróleo. O petróleo foi misturado ao volume total de solo para simular o plantio em uma área contaminada e para que ficasse à disposição da planta via captação pela raiz em qualquer parte do substrato (OECD 2006). As dosagens aplicadas foram: 0 (controle), 0,014, 0,028, 0,056, 0,116 e 0,231 L óleo $\mathrm{m}^{-2}$ de solo.

A cada sete dias foram monitorados o surgimento de brotos e o número destes em cada unidade amostral, já que as plantas possuem capacidade de produzir múltiplos brotos por nó (Piedade 1993). Foi monitorado também o número de folhas normais e com comprometimento fitossanitário (folhas com menos de $30 \%$ da área verde) e o comprimento das folhas das plantas que rebrotaram. Após 63 dias foi obtida a biomassa (massa seca), que foi separada em biomassa aérea viva, biomassa aérea morta, biomassa radicular e a biomassa do colmo. A biomassa total foi calculada pela soma da biomassa aérea viva; biomassa aérea morta e biomassa das raízes.

Tabela 1. Valores de temperatura média (T), temperatura média máxima (TM), temperatura média mínima (Tm), umidade relativa do ar (UR), precipitação total (Pp) e número de dias de chuva (Dc) no ano de 2006 em Manaus (Fonte: Tutiempo.net).

Table 1. Values of average temperature (T), maximum mean temperature (TM), average minimum temperature (Tm), relative humidity (UR), precipitation (Pp) and number of rainy days (Dc) in 2006 in Manaus (Source: Tutiempo.net).

\begin{tabular}{lcccccc}
\hline Período & $\mathbf{T}\left({ }^{\circ} \mathbf{C}\right)$ & $\mathbf{T M}\left({ }^{\circ} \mathbf{C}\right)$ & $\mathbf{T m}\left({ }^{\circ} \mathbf{C}\right)$ & $\mathbf{U R}(\%)$ & Pp $(\mathbf{m m})$ & $\mathbf{D c}$ \\
\hline Julho & 28,1 & 32,2 & 25,2 & 75,6 & 145,8 & 5 \\
Agosto & 28,8 & 32,9 & 25,5 & 72,2 & 11,43 & 2 \\
Setembro & 29,3 & 34,1 & 25,9 & 70,4 & 8,13 & 3 \\
Outubro & 29,7 & 34,2 & 26,3 & 70,2 & 62,49 & 4 \\
A* & 28,45 & 32,55 & 25,35 & 73,9 & 157,23 & 7 \\
& $( \pm 0,35)$ & $( \pm 0,35)$ & $( \pm 0,15)$ & $( \pm 1,7)$ & & \\
B* & 29,5 & 34,1 & 26,1 & 70,3 & 70,62 & 7 \\
& $( \pm 0,2)$ & $( \pm 0,1)$ & $( \pm 0,2)$ & $( \pm 0,1)$ & & \\
\hline
\end{tabular}

*valores médios \pm desvio padrão para o período. 
As unidades amostrais consistiram em 120 frascos distribuídos em um experimento fatorial inteiramente casualizado $2 \times 6$ (períodos $\times$ dosagens de petróleo) com 10 réplicas para cada tratamento. $\mathrm{O}$ efeito da temperatura foi medido por meio da categorização em dois períodos (A e B). As análises estatísticas foram realizadas utilizando o programa Systat 10.2. O efeito da dosagem de petróleo e do período de plantio sobre as variáveis dependentes foi analisado utilizando Análise de Variância bifatoral, respeitando-se os pressupostos de homogeneidade de variâncias e de normalidade. $\mathrm{O}$ teste de Tukey a posteriori foi utilizado para avaliar diferenças estatísticas individuais entre os níveis dos tratamentos $(\mathrm{p}<0,05)$.

\section{Resultados}

Ocorreu uma alta taxa de rebrotamento dos propágulos nos controles, sendo $90 \%$ no período "A" e $100 \%$ no período "B", havendo nos tratamentos com petróleo uma diferença na taxa de
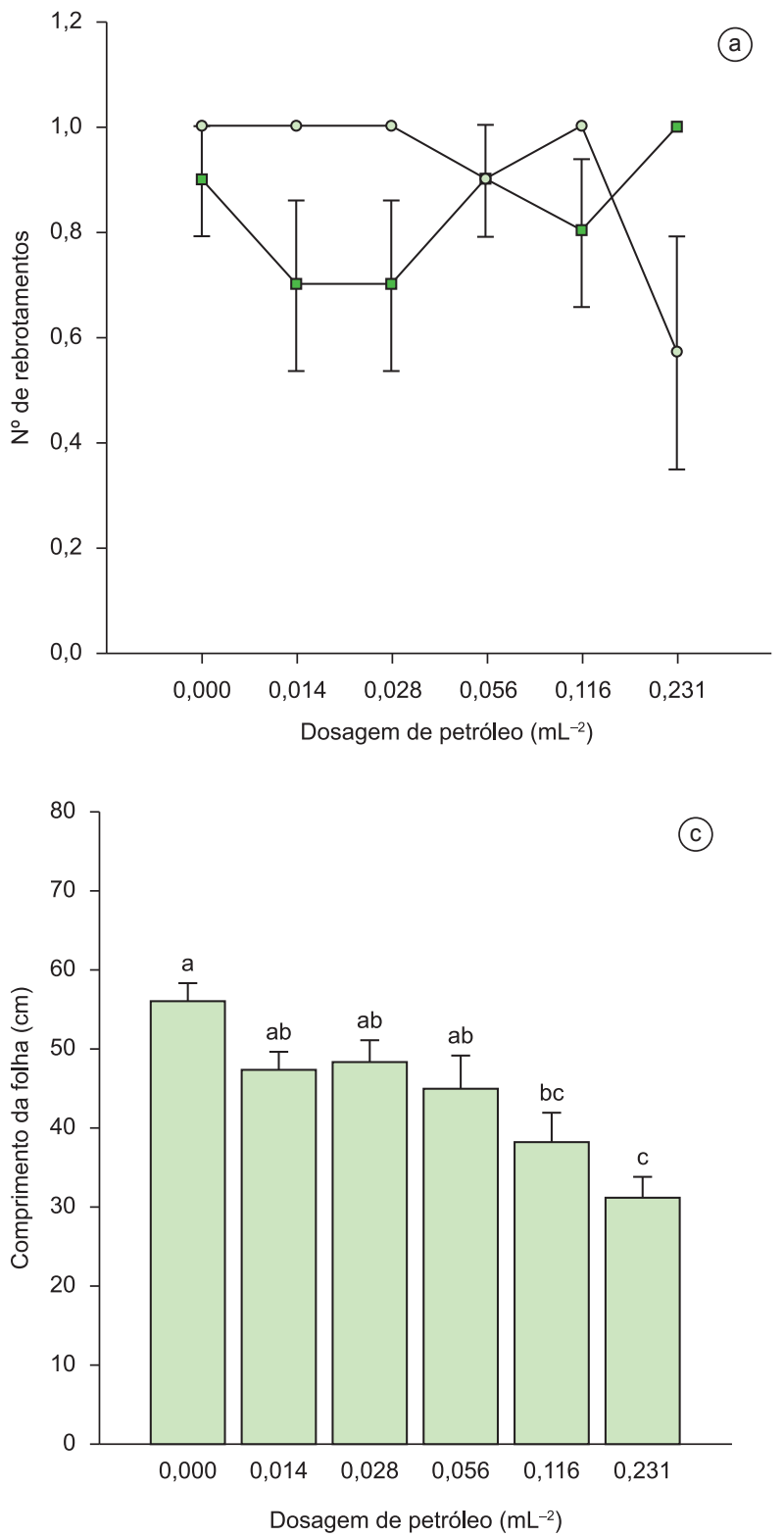

rebrotamento de acordo com o período. No tratamento com dosagem de 0,231 L óleo $\mathrm{m}^{-2}$ de solo houve $100 \%$ de rebrotamento no período "A", e apenas $60 \%$ no período "B". A interação entre a dosagem de petróleo e o período de plantio dos propágulos resultou em uma diminuição do número de rebrotamentos ( $p=0,006$; Figura 1a). $\mathrm{O}$ número de folhas foi influenciado pelo período de plantio dos propágulos ( $p=0,003)$, e embora fosse maior no período "B”, seguiu a mesma tendência de diminuição com o aumento da dosagem de petróleo ( $p=0,006$; Figura 1b). O período de plantio dos propágulos não influenciou o comprimento das folhas $(p>0,05)$. O comprimento das folhas foi reduzido significativamente pelo efeito da dosagem de petróleo $(p<0,0001)$. Os tratamentos com dosagens de $0,116 \mathrm{e}$ $0,231 \mathrm{~L}$ óleo $\mathrm{m}^{-2}$ apresentaram médias significativamente menores para o comprimento das folhas em relação ao controle (Figura 1c).

A biomassa aérea foi afetada pelo petróleo, havendo uma redução com o aumento da dosagem $(p<0,0001)$. Houve influência também
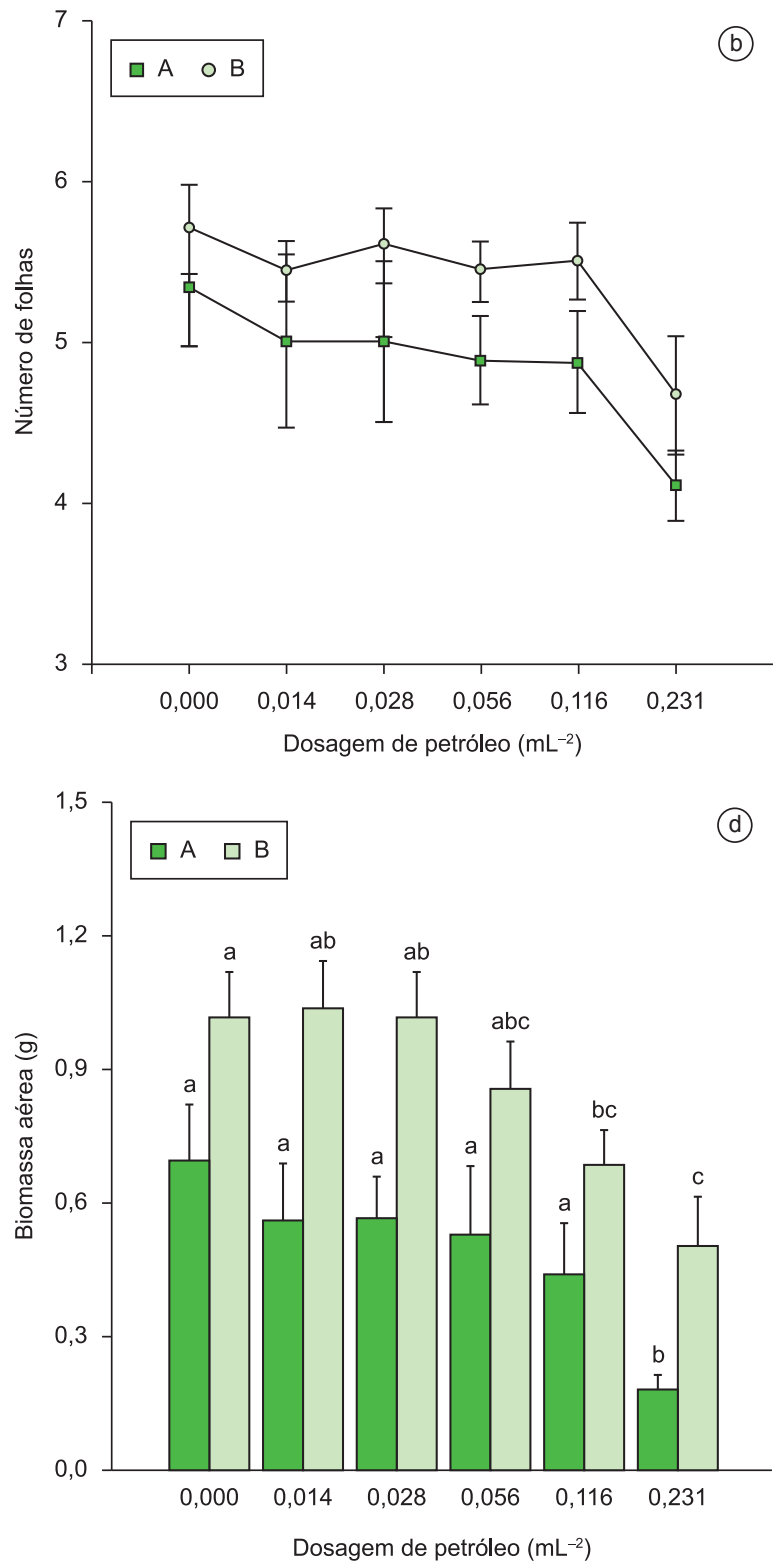

Figura 1. Efeito da dosagem do petróleo sobre E. polystachya: a) número de rebrotamentos; b) número de folhas; c) comprimento da folha, d) biomassa aérea. Períodos de plantio: A (jul./set.), e B (set./nov.). Valores médios com erro padrão.

Figure 1. Effect of oil concentration on E. polystachya: a) number of sprouts; b) number of leaves; c) leaf length; and d) aboveground biomass. Periods of planting: A (July / Sept.), and B (Sept. / Nov.). Mean values with standard error. 
do período de plantio ( $p<0,0001)$, sendo que no período "A" a biomassa aérea média foi menor em todos os tratamentos quando comparado ao período " $\mathrm{B}$ " (1,77 vezes menor no tratamento controle e 2,77 vezes no tratamento de $0,231 \mathrm{~L} \cdot \mathrm{m}^{-2}$ ). No período "A", a biomassa aérea na dosagem de $0,231 \mathrm{~L}$ óleo $\mathrm{m}^{-2}$ de solo foi significativamente menor que o controle, já no período "B" houve redução significativa da biomassa aérea nos tratamentos com dosagens de 0,116 e 0,231 L óleo $\mathrm{m}^{-2}$ de solo em relação ao controle (Figura 1d). Não houve efeito da dosagem de petróleo sobre a produção de biomassa aérea morta $(p<0,05)$, embora tenha ocorrido um efeito significativo do período de plantio dos propágulos $(p<0,0001)$. A biomassa aérea morta foi maior nas plantas no período "B", com uma média de $0,3 \mathrm{~g}$ quando somados todos os tratamentos, enquanto que no período "A" esse valor foi de apenas 0,16 g (Figura 2a). Diferenças significativas entre os períodos também foram encontradas para a biomassa da raiz ( $p<0,0001$; Figura $2 \mathrm{~b}$ ) e do colmo ( $p=0,001$; Figura 2c). Já a biomassa total foi afetada tanto pela dosagem de petróleo $(p=0,018)$ quanto pelo período de plantio $(p<0,0001$; Figura 3a). Quando analisado cada período separadamente, não se detectou efeito significativo da dosagem, ainda que no período de "B" possa ser observada uma tendência $\left(\mathrm{F}_{(6,53)}=2,217, \mathrm{p}=0,056\right)$ de redução da biomassa com o aumento da dosagem de petróleo. Como a proporção de biomassa aérea viva/biomassa aérea total não foi afetada pelo período de plantio, esse fator foi desconsiderado para a análise do efeito da dosagem (Figura 3b). Apenas o tratamento com

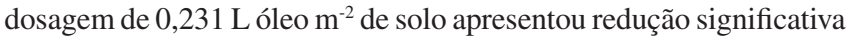
deste parâmetro em relação ao controle $(p<0,0001)$.

\section{Discussão}

O efeito do petróleo em E. polystachya foi influenciado tanto pela dosagem, como pelo período de plantio. A espécie se mostrou muito sensível ao petróleo apresentando alterações nas diversas variáveis analisadas. A capacidade de um organismo responder seja pela alteração na abundância ou biomassa indica o ponto crítico de um poluente (Vandecasteele et al. 2004). Nesse sentido, E. polystachya é uma espécie chave para indicar a integridade dos ambientes sujeitos à contaminação por petróleo nas várzeas do rio Amazonas, por ser uma das espécies mais produtivas e abundantes ao longo dessas planícies inundáveis, e por responder rapidamente à adição de petróleo com aumento da mortalidade (Lopes \& Piedade 2009).
No presente estudo, os propágulos foram plantados logo após o petróleo ter sido misturado ao solo, e as rebrotas levaram entre 2 e 21 dias para aparecer. A mistura do petróleo ao substrato provavelmente facilitou a eliminação de uma considerável parte dos componentes tóxicos por volatilização, reduzindo a toxicidade para as plantas quando as raízes entraram em contato com o solo (Lopes \& Piedade 2009). Além disso, o estoque de nutrientes do colmo pode ter proporcionado às plantas os elementos necessários para o seu desenvolvimento, diminuindo a função de absorção das raízes, diminuindo, consequentemente a absorção de elementos tóxicos presentes no petróleo. Portanto, diferentemente de experimentos prévios realizados com E. polystachya, nos quais a adição de petróleo foi feita na superfície do solo (Lopes et al. 2009), as plantas neste estudo, aparentemente, foram menos afetadas quando o petróleo foi misturado ao volume total do solo antes do plantio dos propágulos. Segundo Lin \& Mendelssohn (1998) os efeitos dos hidrocarbonetos do petróleo na vegetação muitas vezes tendem a diminuir, se a exposição das plantas ao óleo ocorre após um período de tempo, pois o óleo é degradado, principalmente devido à evaporação, dissolução, oxidação e biodegradação, reduzindo seus componentes tóxicos. Após serem eliminados os componentes de baixo peso molecular, mais tóxicos às plantas, são degradados os hidrocarbonetos de peso molecular mais alto, diminuindo gradativamente a toxicidade do óleo remanescente. Esta degradação do óleo ao longo do tempo permite que plantas que sejam sensíveis imediatamente ao poluente possam se desenvolver no solo contaminado, após determinado período de tempo.

O período de plantio dos propágulos ("A" ou "B") mostrou influenciar as diversas variáveis analisadas. A biomassa total sofreu redução devido ao aumento da dosagem de petróleo, havendo um declínio da proporção de biomassa aérea viva/total no tratamento de $0,231 \mathrm{~L}$ em relação ao controle. A biomassa aérea foi afetada pela dosagem de petróleo nos dois períodos de plantio. No período "B" houve redução de $54,03 \%$ da biomassa aérea do tratamento de $0,231 \mathrm{~L}$ em relação ao controle, enquanto que no período "A" houve a redução de $18,86 \%$ neste mesmo tratamento em relação ao controle. O período "B" apresentou ainda redução da biomassa aérea de $84,74 \%$ na dosagem de 0,116 L. Apesar da dosagem do petróleo ter influenciado negativamente o incremento de biomassa pelas plantas em ambos os períodos, no período de temperaturas mais elevadas esse efeito foi mais severo.
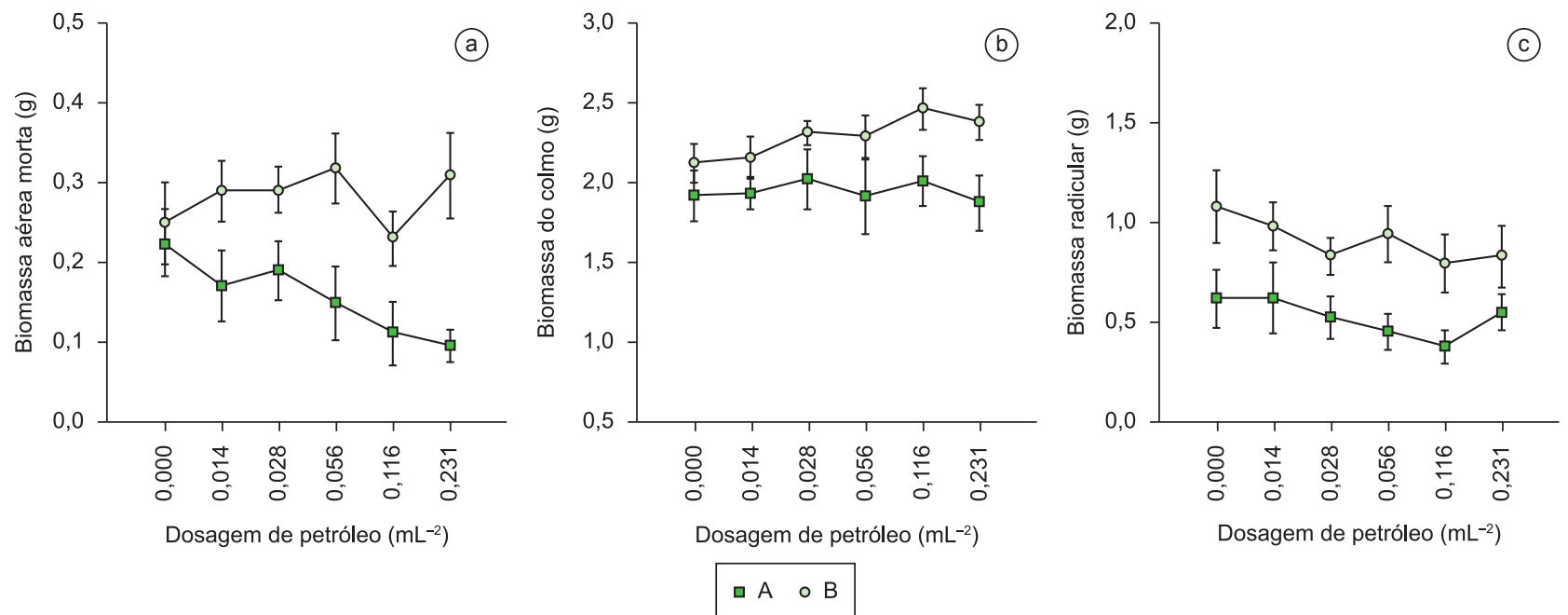

Figura 2. Efeito da dosagem do petróleo e do período de plantio de E. polystachya, A (jul./set.) e B (set./nov.) sobre: a) biomassa aérea morta; b) biomassa da raiz; e c) biomassa do colmo. Valores médios com erro padrão.

Figure 2. Effect of oil concentration and period of planting E. polystachya, A (July / Sept.) and B (Sept. / Nov.) on: a) aboveground dead biomass; b) root biomass; and c) stem biomass. Mean values with standard error. 

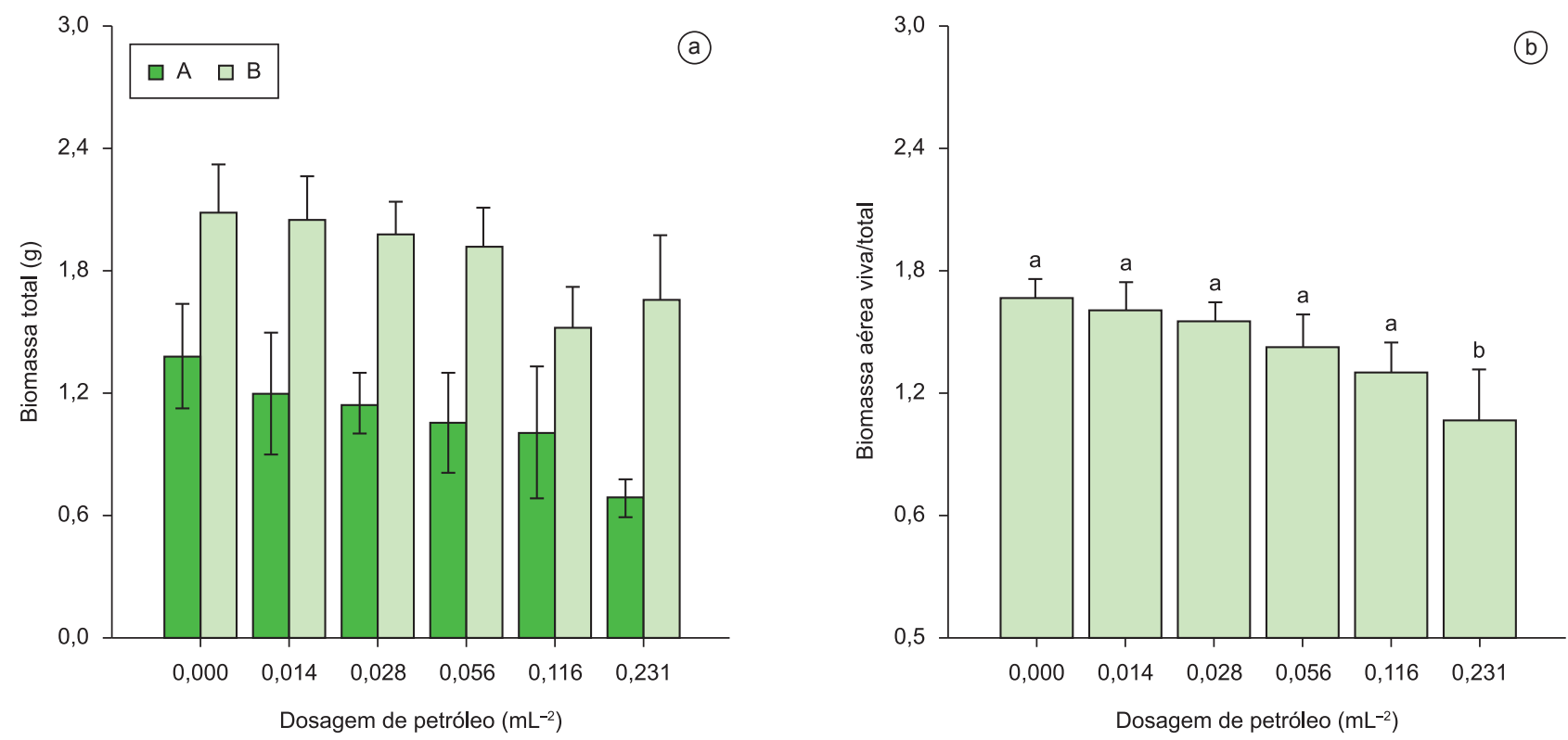

Figura 3. Efeito da dosagem do petróleo sobre: a) biomassa total, nos dois períodos de plantio dos propágulos, A (jul./set.) e B (set./nov.); e b) proporção de biomassa aérea viva/ aérea total de E. polystachya. Valores médios com erro padrão. Letras distintas indicam tratamentos significativamente diferentes $(\alpha=0,05)$.

Figure 3. Effect of oil concentration on: a) total biomass in both periods of planting the propagules, A (July / Sept.) and B (Sept. / Nov.); and b) proportion of live aerial biomass / total aerial biomass of E. polystachya. Mean values with standard error. Different letters indicate significantly different treatments $(\alpha=0.05)$.

Considerando a estratégia reprodutiva de E. polystachya, caracterizada pela perenização por meio da rebrota durante a fase terrestre, fica claro o papel fundamental do colmo para a propagação vegetativa no ambiente natural. Contudo, para efeito deste experimento, no qual o cultivo de E. polystachya foi realizado em casa de vegetação, foram excluídos vários fatores que poderiam influenciar a resposta das plantas, dentre eles: 1) ausência de entrada de água da chuva, ou seja, de variação de precipitação entre os períodos; 2) as pequenas variações de umidade relativas foram excluídas pela irrigação diária das plantas evitando com isso também o estresse hídrico; 3) as mudanças de nível de alagamento às quais as plantas estariam expostas em campo foram excluídas; 4) o solo da várzea é reconhecidamente rico em nutrientes; e 5) a concentração de elementos químicos é muito semelhante entre os talos da planta (Piedade et al., 1992), não existindo portanto efeito da parte do colmo que foi plantada. Assim, dentre os fatores climáticos que poderiam explicar a diferença de resposta das plantas, o único que não foi controlado e apresentou uma variação significativa entre os períodos foi a temperatura (Tabela 1). Houve uma diferença nas temperaturas médias entre estes dois períodos em 2006, de valor pouco superior a $1^{\circ} \mathrm{C}$, porém, quando as médias das temperaturas máximas e mínimas para cada um dos períodos são observadas, percebe-se claramente que no período " $\mathrm{A}$ " elas foram relativamente mais baixas, $32,5^{\circ} \mathrm{C}$ e $28,4^{\circ} \mathrm{C}$, respectivamente, que no período "B", $34,1^{\circ} \mathrm{C}$ e $29,5^{\circ} \mathrm{C}$ atingindo temperaturas de até $34,5^{\circ} \mathrm{C}$ no período " $\mathrm{A}$ " e $36,2^{\circ} \mathrm{C}$ no período "B".

A temperatura é apontada como um agravante do impacto do óleo sobre as respostas das plantas (Bowen 1996; Merkl et al. 2005). Merkl et al. (2005) explicaram as modificações no comprimento e morfologia das raízes de Brachiaria brizantha, Cuperus agrregatus e Eleusiane indica expostas ao óleo cru da Venezuela pelo aumento de temperatura do solo. No presente estudo, o fato do número de brotos por unidade amostral, biomassa total e proporção de biomassa aérea viva/total só sofrerem redução devido ao efeito da dosagem do petróleo no período "B", caracterizado por maiores temperaturas, ilustra a influência negativa desde fator sobre E. polystachya exposta ao petróleo.
Uma das possíveis explicações para o efeito da temperatura sobre o impacto do petróleo é que o aumento de temperatura diminui a solubilidade dos gases na água, ocorrendo assim um decréscimo na quantidade de oxigênio dissolvido na água, diminuído a disponibilidade de oxigênio para as raízes. Outra explicação possível é que solos contaminados por petróleo podem apresentar temperaturas mais altas que solos não contaminados (Merkl et al. 2005). Além disso, o aumento de temperatura é um fator que pode ser imediatamente estressante para algumas espécies de plantas (Taiz \& Zeiger 2004). E, embora temperaturas muito baixas, próximas a $4{ }^{\circ} \mathrm{C}$ inibam a atividade de microrganismos degradadores de hidrocarbonetos (Iqbal et al. 2007), em altas temperaturas a atividade de fungos e bactérias também são reduzidas (Wise \& Torantolo 1994), diminuindo o consumo de hidrocarbonetos que, ficando disponíveis no solo, poderiam ser absorvidos pelas plantas.

O petróleo cru é constituído por uma complexa mistura de componentes orgânicos, sendo que $75 \%$ dele consiste de hidrocarbonetos (Neff 1979). Os hidrocarbonetos de cadeia curta são voláteis possuindo um tempo de vida relativamente curto no ambiente aquático, porém esta é a fração mais tóxica para os organismos aquáticos. Já os hidrocarbonetos de cadeia longa são pouco solúveis podendo persistir por um longo tempo no ambiente; seus efeitos são principalmente físicos, criando uma barreira viscosa na interface água-ar (Val \& Almeida-Val 1999). A viscosidade, resistência oferecida por qualquer fluido ao movimento ou escoamento, é inversamente proporcional à temperatura. Portanto, em temperaturas mais elevadas o petróleo ficaria menos viscoso e a sua absorção pelo solo poderia ocorrer de forma mais rápida, fazendo com que a camada superficial de óleo entrasse em contato com as raízes. No presente estudo a biomassa da raiz no tratamento de $0,231 \mathrm{~L}^{\text {óleo }} \mathrm{m}^{-2}$ de solo foi $50,61 \%$ menor no período "B" que no período "A". Além disso, apesar de não ter apresentado alteração significativa pelo aumento da dosagem de petróleo, a biomassa do colmo de E. polystachya foi maior no período "B" que no período "A", o que pode indicar um menor aproveitamento de suas reservas pelas plantas na fase inicial de desenvolvimento que ocorre durante este período. 


\section{Conclusão}

Doses mais altas do petróleo, como 0,231 L óleo $\mathrm{m}^{-2}$ de solo foram limitantes para o estabelecimento de E. polystachya. A comparação dos indivíduos de E. polystachya que foram plantados em diferentes períodos ("A" e "B") mostrou que a resposta dessa espécie é influenciada pela temperatura, sendo que todos os parâmetros mensurados indicam que temperaturas mais elevadas (período "B") maximizam os efeitos negativos do petróleo sobre esta espécie. Podese concluir que a sazonalidade é um fator muito importante também nos trópicos e deve ser considerada nos experimentos de avaliação da fitotoxicidade do petróleo.

\section{Agradecimentos}

Os recursos necessários foram providos pelo Projeto INPA/MaxPlanck, PPI 1090-5. O petróleo foi cedido pela Petrobrás através do Projeto PIATAM. Agradecemos ao CNPq pela Bolsa de Mestrado de Aline Lopes, ao Projeto INPA/Max-Planck e ao Laboratório de Ecofisiologia e Evolução (LEEM) pelo apoio técnico e logístico. Ao Centro de Estudos Superiores do Trópico Úmido da Universidade do Estado do Amazonas agradecemos pela bolsa PCI cedida a Aline Lopes durante a elaboração do artigo.

\section{Referências Bibliográficas}

AGÊNCIA NACIONAL DO PETRÓlEO, GÁS NATURAL E BIOCOMBUSTÍVEIS - ANP. 2008. Despacho do Diretor-Geral $\mathrm{n}^{\circ}$ 683, Anexo II: relação de tipos de petróleo nacional, p.13.

BOWEN, G.D. 1996. Soil temperature, root growth and plant function. In Plant Roots: the hidden half (Y. Waisel, U. Eshel \& U. Kafkafi, eds.). Marcel Dekker, New York, p.309-329.

CUNNINGHAM, S.D., BERTI, W.R. \& HUANG, J.W. 1995. Phytoremediation of contaminated soils. Trends Biotechnol. 13:393-397.

DINARDI, A.L., FORMAGI, V.M., CONEGLIAN, C.M. R., BRITO, N.M. DE, SOBRINHO, G.D., TONSO, S. \& PELEGRINI, R. 2003. Fitorremediação. In Anais do III Fórum de Estudos Contábeis, Faculdades Integradas Claretianas, Rio Claro, Brasil.

DOWTY, R.A., SHAFFER, G.P., HESTER, M.W., CHILDERS, G.W., CAMPO, F.M. \& GREENE, M.C. 2001. Phytoremediation of small-scale oil spills in fresh marsh environments: a mesocosm simulation. Mar. Environ. Res. 52: 195-211.

GLICK, B.R. 2003. Phytoremediation: synergistic use of plants and bacteria to clean up the environment. Biotechnol. Adv. 21:383-393.

IQBAL, J., METOSH-DICKEY, C. \& PORTIER, R.J. 2007. Temperature effects on bioremediation of PAHs and PCP contaminated south Louisiana soils: a laboratory mesocosm study. J. Soils Sediments. 7(3):153-158.

JUNK, W.J., BAYLEY, P.B. \& SPARKS, R.E. 1989. The flood pulse concept in river-floodplain systems. In: Proceedings of the International Large River Symposium (Dodge DP ed.). Ottawa: Can. Spec. Publ. Fish. Aquat. Sci., p.110-127.

LIN, Q. \& MENDELSSOHN, I.A. 1998. The combined effects of phytoremediation and biostimulation in enhancing habitat restoration and oil degradation of petroleum contaminated wetlands. Ecol. Eng.10:263-274.

LOPES, A. 2007. Respostas de herbáceas aquáticas amazônicas ao petróleo cru de Urucu (Coari - AM). Dissertação de Mestrado, Instituto Nacional de Pesquisas da Amazônia, Universidade Federal do Amazonas, Manaus.

LOPES, A., PIEDADE, M.T.F. \& ALMEIDA-VAL, V.M.F. 2005. Efeito da dosagem do petróleo de Urucu (Coari-AM) sobre a sobrevivência de Echinochloa polystachya (Projeto Piloto). In Anais do I Congresso Internacional do PIATAM. Universidade Federal do Amazonas / Centro de Pesquisas da Petrobrás, Manaus, p.113-113.

LOPES, A. \& PIEDADE, M.T.F. 2009. Estabelecimento de Echinochloa polystachya (H.B.K.) Hitchcock (Poaceae) em solo de várzea contaminado com petróleo de Urucu. Acta Amazon.39(3): 583-590.

LOPES, A., ROSA-OSMAN, S. \& PIEDADE, M.T.F. 2009. Effects of crude oil on survival, morphology, and anatomy of two aquatic macrophytes from the Amazon floodplains. Hydrobiologia 636(1): 295-305.

MERKL, N., SCHLTZE-KRAFT, R., INFANTE, C. 2005. Phytoremediation in the tropics: influence of heavy crude oil on root morphological characteristics of graminoids. Environ. Pollut. 138:86-91.

NEFF, J.M. 1979. Polycyclic aromatic hydrocarbons in the aquatic environment: sources, fates and biological effects. Applied Science Publishers, Essex.

PETROBRAS. 2007. Balanço social e ambiental 2007. Petrobras, Rio de Janeiro.

PEZESHKI, S.R., HESTER, M.W., LIN, Q. \& NYMAN, J.A. 2000. The effect of oil spill and cleanup on dominant US Gulf coast marsh macrophytes: a review. Environ. Pollut. 180: 129-139.

PIEDADE, M.T.F. 1988. Biomassa, produtividade e atividade fotossintética de Echinochloa polystachya (H.B.K.) Hitchcock (Gramineae = Poacea), capim semi-aquático da várzea amazônica. Tese de Doutorado, Universidade Federal do Amazonas, Manaus, Amazonas.

PIEDADE, M.T.F. 1993. Biologia e ecologia de Echinochloa polystachya (H.B.K) Hitchcock (Gramineae=Poaceae), capim semi-aquatico da várzea amazônica. Acta Limnol.Bras. 4:173-185.

PIEDADE, M.T.F., JUNK, W.J. \& LONG, S.P. 1991. The productivity of the C4 grass Echinochloa polystachya on the Amazon floodplain. Ecology 72(4):1456-1463.

PIEDADE, M.T.F., JUNK, W.J. \& MELLO, J.A.S.N. 1992. A floodplain grassland of the Central Amazon. In Productivity of grass ecosystems of the Tropics and Sub-Tropics (S.P. Long, M.B. Jones \& J.M. ROBERTS, eds.) Chapman \& Hall, London, p.127-158.

PIEDADE, M.T.F., JUNK, W.J. \& LONG, S.P. 1997. Nutrients dynamics of the highly productive $\mathrm{C} 4$ macrophyte Echinochloa polystachya on the Amazon floodplain. Functional Ecology 11: 60-65.

SCHUSSLER, E.E. \& LONGSTRETH, D.J. 1996. Aerenchyma develops by cell lysis in roots and cell separation in leaf petioles in Sagittaria lancifolia (Alismataceae). Am J Bot. 83: 1266-1273.

TAIZ, L. \& ZEIGER, E. 2004. Fisiologia Vegetal terceira ed. Atmed, Porto Alegre.

TUTIEMPO.NET. Historical weather: Manaus Aeroporto, year 2006. http:// www.tutiempo.net (último acesso em 01/03/2007).

VAL, A. L. \& ALMEIDA-VAL, V.M. 1999. Effects of crude oil on respiratory aspects of some fish species of the Amazon. In Biology of Tropical Fishes (A.L. Val \& V.M.F. Almeida-Val, eds.). INPA, Manaus.,p.277-291.

VANDECASTEELE, B., SAMYN, J., QUATAERT, P., MUYS, B. \& TACK, F.M.G. 2004. Earthworm biomass as additional information for risk assessment of heavy metal biomagnification: a case study for dredged sediment-derived soils and polluted floodplain soils. Environ. Pollut. 129:363-375.

WISE, D.L. \& TORANTOLO, D.J. 1994. Remediation engineering of hazardous waste contaminated soil. Marcel Dekker, NY.

ZENGEL, S.A. \& MICHEL, J. 1996. Vegetation cutting as a clean-up method for salt and brackish marshes impacted by oil spills: a review and case history of the effects on plant recovery. Mar. Pollut. Bull. 32(12): 876885 . 